\title{
Report on the 1st Balkan Nephrology School held in Prishtina/Kosova and on the first living related kidney transplantation at the University Hospital of Prishtina, 23-26 May 2007
}

\author{
Nexhat Miftari ${ }^{1}$, Jörg Beimler ${ }^{1}$, Christian Morath ${ }^{1}$, Jan Schmidt ${ }^{2}$ and Martin Zeier ${ }^{1}$ \\ ${ }^{1}$ Department of Medicine, Division of Nephrology and ${ }^{2}$ Department of Visceral- and Transplantation Surgery University \\ of Heidelberg, Heidelberg, Germany
}

\section{Introduction}

In the past 6 years, various efforts have been made to improve the treatment of patients with renal disease in Kosova. In June 2000, two WHO-appointed consultants wrote an extended report on future needs, to guarantee that end-stage renal disease (ESRD) patients in Kosova are treated to general standards [1]. This was followed in 2001 by a group of six people who were sent by the ISN Commission on Acute Renal Failure and the Joint Action Eastern Europe (ISN/ERA), to support the renal unit with technical advice, to give teaching lessons and to help improve dialytic treatment immediately after the war. The ISN/ERA group met staff members and trainees of the Department of Nephrology and worked with them for an entire week [2]. In 2002, another short visit was initiated by the ISN/EDTA; this was followed by a private initiative of the University of Heidelberg for nephrology seminars for trainees and young nephrologists in 2005 and 2006. Attendance and interest in both meetings were extraordinarily high and a report for ISN and EDTA was filed. Based on this report, ISN and EDTA officially supported the 2007 Balkan Nephrology School, which was held by members of the Renal Divisions of the Universities of Vienna, Ghent and Heidelberg.

\section{Balkan nephrology school and the first living-related renal transplantation}

This ISN/EDTA sponsored Nephrology School attracted 120 physicians from Kosova, Albania, Bosnia, Montenegro and Macedonia (FYROM).

Correspondence to: Martin Zeier, MD, Department of Medicine Nephrology, University of Heidelberg, Im Neuenheimer Feld 162, 69120 Heidelberg. Email: martin.zeier@med.uni-heidelberg.de
The attendees were in training for internal medicine or in sub-speciality training as nephrologists.

The Nephrology School covered clinical nephrology topics, such as urinary sediment and ultrasound findings, the diagnosis and treatment of glomerular diseases, e.g. diabetic nephropathy and rapidly progressive glomerulonephritis, and the concepts of retarding the progression of chronic kidney disease (CKD). The basic principles of haemodialysis therapy were discussed, with an introduction to the concept of dry weight, blood pressure control on dialysis, the creation and monitoring of arteriovenous fistula and hygiene standards, with special emphasis on dialysis water supply. The diagnosis of acute renal injury and the concomitant treatment of acute renal failure was another topic during the 1st Balkan Nephrology School. The prevalence of ESRD is 285 patients per 1000000 population in Kosova and consumes a considerable amount of the health care budget. The initiation of a peritoneal dialysis (PD) programme was therefore another important part of the teaching sessions. The programme organizers felt that a teaching programme and an algorithm to recruit PD patients were important. The principles and practice of $\mathrm{PD}$, the monitoring of $\mathrm{PD}$ adequacy and the diagnosis and treatment of complications were extensively discussed.

The principles and practice of living related kidney transplantation and the long-term treatment of kidney allograft recipients were covered. This was of particular note, since the transplantation team of Prof. Jan Schmidt performed the first living related kidney transplantation in Kosova, where the right kidney of a mother was transplanted to her 15 -yearold daughter, with immediate function and no post-operative complications. She received a standard immunosuppression with methylprednisolone, ciclosporin A and mycophenolic acid. Since the majority of haemodialysis patients in Kosova are younger than 60 years of age, which is quite different to Western Europe, we believe that living related 
transplantation in a tertiary centre, is a treatment option for ESRD patients in Kosova. The Balkan Nephrology School is therefore an option to teach local nephrologists how to manage renal transplant recipients in long-term follow-up. Graft monitoring and the reading of renal biopsies was discussed with Prof. Regele, a nephropathologist from Vienna. He provided the basic information to set up a renal pathology unit and to exchange biopsy findings. The participants were actively involved in discussions and an intensive dialogue between faculty and participants during the whole Balkan Nephrology School was noted.

In summary, the 1st Balkan Nephrology School held by the Renal Divisions of the Universities of
Ghent, Heidelberg and Vienna was very successful and warrants further training sessions in the future.

Conflict of interest statement. None declared.

\section{References}

1. Brown W, Adams M. Assessment of the care of renal patients in Kosova. 2000. (Report available at: Kim.Solet@Ualberta.CA)

2. Claus S, de Roose J, Lemaire J, Spatzker S, Veys N, Zeier M. Report on Training Sessions at the Prishtina University Hospital Department of Nephrology and Dialysis Unit, 22-29 July 2000 by the Joint Action Nephrology Eastern Europe of ISN and EDTA/ERA

Received for publication: 14.6.07

Accepted in revised form: 15.6.07 